\section{Estratégias de enfrentamento, dificuldades funcionais e fatores associados em idosos institucionalizados}

\author{
Coping strategies, functional difficulties, and \\ associated factors in institutionalized elderly
}

1 Pontifícia Universidade Católica de Porto Alegre, Porto Alegre, Brasil.

Correspondência A. S. Vivan

Pontifícia Universidade Católica de Porto Alegre. Av. Protásio Alves 658 apto. 03, Porto Alegre, RS 90410-004, Brasil. anavivan@uol.com.br

\begin{abstract}
The aim of this study was to analyze the strategies used by elderly people to cope with functional difficulties, and to investigate a possible association between such strategies and variables like depressive symptoms, gender, schooling, marital status, and self-perceived health. 103 institutionalized elderly individuals participated in the study. They required assistance for at least one activity of daily living. The study was cross-sectional and used the following indicators: Personal Data Chart, Activities of Daily Living Scale, Modified Mini International Neuropsychiatric Interview, Mini-Mental Examination, Coping Strategies Inventory, and Geriatric Depression Scale. The most widely used coping strategies in the sample were self-control and problem solving. Significant associations were found between schooling and depression. The findings suggest that assessing coping strategies for stressful events can assist analysis of the situation and appropriate adaptation of the chosen strategies, so as to foster changes within the medical context.
\end{abstract}

Health of Institutionalized Elderly; Depression; Frail Elderly
Analise de Souza Vivan ${ }^{1}$

Irani Iracema de Lima Argimon 1

\section{Introdução}

De acordo com dados do Instituto Brasileiro de Geografia e Estatística (IBGE) 1, a população de idosos vem aumentando nos últimos anos. Estudos apontam uma queda da natalidade e um número crescente de idosos. No ano de 2000, 30\% da população estava situada na faixa etária de 0 a 14 anos, enquanto os maiores de 65 anos representavam $5 \%$ dos brasileiros. A estimativa é de que, em 2050, esses dois grupos se igualem, representando, cada um, 18\% da população ${ }^{2}$. Em face disso, muitos pesquisadores têm se interessado pelo envelhecimento humano, estimulando uma multiplicidade de estudos e avaliações sobre a velhice.

Busse \& Blazer 3 conceituam o termo envelhecer como as alterações físicas que se desenvolvem na idade adulta, resultando em um declínio na eficiência do funcionamento. O sentimento de controle e a ausência de seqüelas ou incapacidades associadas pode ser o que contribui para os idosos expressarem satisfação em relação à vida. Porém, quando eles deparam com a falta de autonomia e a necessidade de assistência, ou dependência nas atividades da vida diária, a qualidade de vida parece estar em risco 4 .

Capacidade funcional vem sendo vista como um novo paradigma de saúde para o idoso. A presença de uma doença, falta de autonomia, fatores culturais, sócio-econômicos e estilo de vida podem comprometer a capacidade funcional de 
um indivíduo, afetando o bem-estar e o processo de envelhecimento saudável 5,6,7.

Em pesquisa desenvolvida por Silva ${ }^{8}$, em que se verificaram as vantagens e desvantagens da velhice, houve uma grande ênfase nos fatores de saúde, principalmente no que implica limitações e prejuízos no dia-a-dia. A possibilidade de executar as atividades cotidianas sem necessitar de interferência ou influência de outras pessoas está relacionada com a percepção de qualidade de vida 9 .

Um estudo desenvolvido no Brasil estimou que $10 \%$ dos idosos necessitam de assistência nas atividades de vida diária, enquanto $40 \%$ precisam de ajuda nas atividades instrumentais de vida diária 10 . No entanto, esses números tendem a apresentar significativa elevação em estudos desenvolvidos com idosos residentes em instituições asilares. Guedes \& Silveira 11 investigaram 109 idosos neste caso e constataram que $40,36 \%$ deles necessitavam de assistência ou eram dependentes nas atividades de vida diárias. Na análise individual de cada atividade, o mais elevado índice de idosos dependentes situou-se na atividade de banho, totalizando $67,89 \%$. Pesquisa similar desenvolvida com 39 idosos institucionalizados constatou, através do índice de Katz, que 51,35\% dos idosos foram considerados independentes e 48,65\%, dependentes ou necessitados de assistência nas atividades de vida diária 12 .

A situação de dificuldade funcional e a necessidade de assistência nas atividades de vida diária e nas atividades instrumentais de vida diária podem representar um fator estressante no processo de envelhecimento. $\mathrm{O}$ aparecimento progressivo de doenças e dificuldades funcionais são fatores determinantes da incidência do estresse na terceira idade 13 .

Na concepção de Lazarus \& Folkman 14, o estresse é resultado da relação entre a pessoa e o ambiente. Os autores introduzem o termo coping referindo-se ao conjunto de esforços, cognitivos e comportamentais, utilizado pelos indivíduos com o objetivo de lidar com demandas específicas, protegendo-os de aspectos considerados ameaçadores ao seu bem-estar. Coping é um processo dinâmico, que pode estar em constante mudança, de acordo com as reavaliações feitas sobre o evento, e pode ter como conseqüência resultados melhores ou piores do que a situação inicial.

As estratégias de coping são classificadas em dois tipos: focalizada na emoção e focalizada no problema. O primeiro tem como objetivo alterar o estado emocional do indivíduo, buscando reduzir a sensação física desagradável do estado de estresse. Já o coping focalizado no problema procura alterar a dificuldade existente na relação entre as pessoas e o ambiente, podendo direcionar sua ação interna ou externamente 15 .

Uma importante variável no estudo de coping, segundo Zeidner \& Saklofske 16, é a depressão, pois, além de interferir na interpretação de uma situação, algumas características depressivas afetam a seleção de estratégias de coping e a percepção real de sua eficácia. Um estudo longitudinal desenvolvido por Kraaij et al. 17, envolvendo coping e depressão, acompanhou 99 idosos por um período de dois anos e meio. Os resultados apontam as principais estratégias de enfrentamento utilizadas pela amostra. Os idosos que apresentaram mais sintomas depressivos utilizaram em maior quantidade as estratégias de aceitação, ruminação e catastrofização, enquanto os sujeitos com menos sintomas depressivos manifestaram mais a reavaliação positiva. $\mathrm{Na}$ avaliação longitudinal, na medida em que os sintomas depressivos eram controlados, as estratégias observadas eram as de aceitação e reavaliação positiva.

Dessa forma, levando-se em consideração os prejuízos na qualidade de vida dos idosos que apresentam dificuldades funcionais, o presente estudo tem como objetivo verificar as estratégias de coping utilizadas pelos participantes e a existência de associação entre o uso das estratégias e variáveis como: sintomas depressivos, sexo, escolaridade, idade, estado civil e percepção da saúde.

\section{Método}

\section{Participantes}

Foi realizada uma amostragem por conveniência, com $95 \%$ de confiança e $10 \%$ de erro máximo. Como critérios de inclusão, os participantes deveriam apresentar necessidade de assistência em, no mínimo, uma atividade de vida diária. Além disso, foram excluídos do estudo idosos com doenças clínicas incapacitantes, demência, presença de transtornos psicóticos e dependência de substâncias psicoativas, excluindo-se nicotina e cafeína. Assim, participaram da pesquisa 103 idosos institucionalizados, com idade acima de 60 anos, de escolaridade e nível sócio-econômico diversos, residentes na cidade de Porto Alegre, Estado do Rio Grande do Sul, Brasil.

\section{Instrumentos}

Para a coleta de dados, foram utilizados os seguintes instrumentos: Ficha de Dados Pessoais, Escala de Atividades da Vida Diária (EAVD), Breve 
Entrevista Internacional de Neuropsiquiatria Modificada (MINI), Mini-Exame do Estado Mental (MMSE), Inventário de Estratégias de Coping e Escala de Depressão Geriátrica (GDS), que serão descritos a seguir.

A Ficha de Dados Pessoais continha itens referentes ao idoso, incluindo idade, sexo, estado civil, tempo de residência em instituição asilar, anos de escolaridade, profissão, proveniência da renda, religião, atividades de lazer e informações sobre saúde.

A EAVD, proposta por Katz 18, foi utilizada para a verificação do estado funcional. Na escala, constam as tarefas de banho, vestuário, higiene pessoal, transferência, continência e alimentação, em que o participante informa se é independente, se necessita de assistência ou se é dependente para a realização da tarefa.

A MINI, utilizada para verificar os critérios de exclusão, consiste em uma breve entrevista estruturada para a triagem dos principais quadros psicopatológicos. Permite a formulação de diagnósticos compatíveis com os critérios do DSM-IV (Manual Diagnóstico e Estatístico dos Transtornos Mentais da American Psychological Association) e da CID-10 (Classificação Internacional de Doenças, 10a revisão, da Organização Mundial da Saúde). A atual versão é utilizada em mais de 30 idiomas, sendo validada no Brasil por Amorim 19.

O MMSE foi utilizado para avaliar as condições intelectuais dos sujeitos e verificar eventuais prejuízos cognitivos. O MMSE, desenvolvido por Folstein et al. 20, é composto por questões agrupadas em sete categorias, planejadas para avaliar funções cognitivas específicas: orientação para tempo (5 pontos), orientação para local ( 5 pontos), registro de três palavras ( 3 pontos), atenção e cálculo (5 pontos), lembrança das três palavras ( 3 pontos), linguagem ( 8 pontos) e capacidade construtiva visual (1 ponto). O escore do MMSE pode variar de um mínimo de zero até um total máximo de 30 pontos, com ponto de corte de 24 .

O Inventário de Estratégias de Coping, proposto por Lazarus \& Folkman 14 , é composto por 66 itens, incluindo pensamentos e ações utilizadas para lidar com demandas internas ou externas de determinado evento estressante. Os autores propõem oito fatores classificatórios, os quais foram reorganizados e mantidos por Savóia et al. 21 após a verificação da confiabilidade e validade à realidade brasileira. São eles: confronto (itens $07,17,28,34,40,47$ ), afastamento (itens $06,10,13,16,21,41,44)$, autocontrole (itens 14, $15,35,43,54$ ), suporte social (itens $08,18,22,31$, 42,45 ), aceitação de responsabilidade (itens 09, $25,29,48,51,52,62$ ), fuga e esquiva (itens 58 ,
59), resolução de problemas (itens 01, 26, 46, 49) e reavaliação positiva (itens 20, 23, 30, 36, 38, 39, $56,60,63)$.

A GDS, desenvolvida por Yesavage et al. ${ }^{22}$, foi utilizada para identificar os sintomas depressivos dos idosos. O instrumento consiste em 15 questões, com alternativas de resposta sim e não, em que escores inferiores a 5 são considerados normais, de 5 a 10 indicam depressão leve a moderada e acima de 10, depressão grave.

\section{Procedimentos}

Foi realizado contato com algumas instituições asilares de Porto Alegre para explicar os objetivos da pesquisa e obter as autorizações necessárias. Os instrumentos foram aplicados individualmente, em salas ou outro local reservado na própria instituição. A coleta de dados foi realizada pela pesquisadora e quatro alunos de graduação, previamente treinados.

A análise quantitativa foi efetivada por meio de estatísticas descritivas, com o objetivo de traçar o perfil sócio-demográfico e fazer o levantamento das estratégias utilizadas. Foram calculadas as médias, desvio padrão, freqüências e percentuais.

Para testar as hipóteses da pesquisa, foi utilizado o teste exato de Fisher. Os dados foram tabulados com base no programa SPSS 11.5 (SPSS Inc., Chicago, Estados Unidos) com o nível de significância de $5 \%$.

O projeto de pesquisa foi aprovado pelo Comitê de Ética da Pontifícia Universidade Católica do Rio Grande do Sul (Porto Alegre, Rio Grande do Sul, Brasil). Todos os participantes do estudo receberam e assinaram o Termo de Consentimento Livre e Esclarecido.

\section{Resultados}

Dos 103 idosos que constituíram a amostra, a idade variou entre 60 e 95 anos, tendo como média 79,66 anos (DP = 9,71). O tempo de residência mínimo em asilo foi de até 1 ano e o máximo de 26 anos, tendo 4,34 anos como média (DP = $4,84)$. A média de escolaridade manteve-se em 6,53 anos ( $\mathrm{DP}=4,34$ ), variando de nenhum ano de escolaridade até o máximo de 15 anos.

Quanto às freqüências relacionadas à amostra, a maioria dos idosos é do sexo feminino $(88,3 \%)$ e viúva $(56,9 \%)$. Em relação à proveniência da renda, 53,4\% são aposentados. No que se refere à percepção da própria saúde, 11,7\% classificaram-na como má. A maioria da amostra $(83,5 \%)$ realiza exames de rotina periodicamente. A religião foi variada, com a maioria católica 
$(60,2 \%)$ e praticante $(81,6 \%)$. A principal atividade de lazer foi a de ver televisão $(70,9 \%)$, seguida de ouvir música $(61,8 \%)$ e de praticar atividades manuais $(50,5 \%)$.

A EAVD que mais obteve dependência por parte dos sujeitos foi a atividade de vestuário $(24,3 \%)$. No entanto, quando analisada a necessidade de assistência juntamente com a dependência, temos a atividade de continência com maior percentual $(49,5 \%)$, seguida de vestuário $(47,6 \%)$ e banho $(43,7 \%)$. A única atividade que não manifestou dependência de nenhum sujeito foi a de alimentação (Figura 1).

Para a análise das estratégias de coping mais utilizadas pelos participantes, foi adotado o critério de avaliação que determina a utilização da estratégia em seus pontos altos (utiliza grande parte das vezes e utiliza quase sempre), considerado na Figura 2 como "utiliza". Para os pontos mais baixos (não utiliza ou utiliza pouco e utiliza algumas vezes), adotou-se a indicação "não utiliza”. Dessa forma, as estratégias mais utilizadas em face de situações de dificuldade funcional, com $44,7 \%$ cada, foram a de autocontrole e a de resolução de problemas, seguidas pela de suporte social, com $39,8 \%$.

Quanto à relação entre as estratégias de coping e as demais variáveis, os resultados obtidos mediante o teste exato de Fisher mostraram que houve associação significativa entre a estratégia de suporte social e os sintomas depressivos $(\mathrm{p}=$
0,027), indicando que quanto menos sintomas depressivos apresentados, mais os idosos utilizam a estratégia de suporte social. Para os idosos que apresentaram sintomas depressivos em nível grave, a estratégia de fuga e esquiva aparece como a mais utilizada (45,5\%); no entanto, não se verificou associação significativa $(p=0,801)$ (Tabela 1).

Em relação ao sexo (Tabela 2), os homens apresentaram maior uso da estratégia de resolução de problemas $(66,7 \%)$, seguida de fuga e esquiva (50\%), enquanto as mulheres demonstraram tendência à utilização da estratégia de autocontrole $(45,1 \%)$, seguida de resolução de problemas e suporte social $(41,8 \%)$. Não foi evidenciada, contudo, associação significativa entre os sexos e as diferentes estratégias de enfrentamento.

Quanto à escolaridade (Tabela 3), os idosos foram classificados em três diferentes grupos: analfabetos, de 1 a 4 anos de escolaridade e 5 anos ou mais de escolaridade. A única das estratégias de enfrentamento que apresentou associação significativa foi o confronto, da qual idosos com 1 a 4 anos de escolaridade apresentaram maior uso $(p=0,001)$.

Para a análise estatística da relação entre idade e estratégias de coping, foram traçadas faixas etárias, compreendendo as idades de 60 a 69 anos, de 70 a 79 anos, de 80 a 89 anos e acima de 90 anos. Não foram obtidos resultados signi-

Figura 1

Atividades da vida diária e necessidades dos idosos.

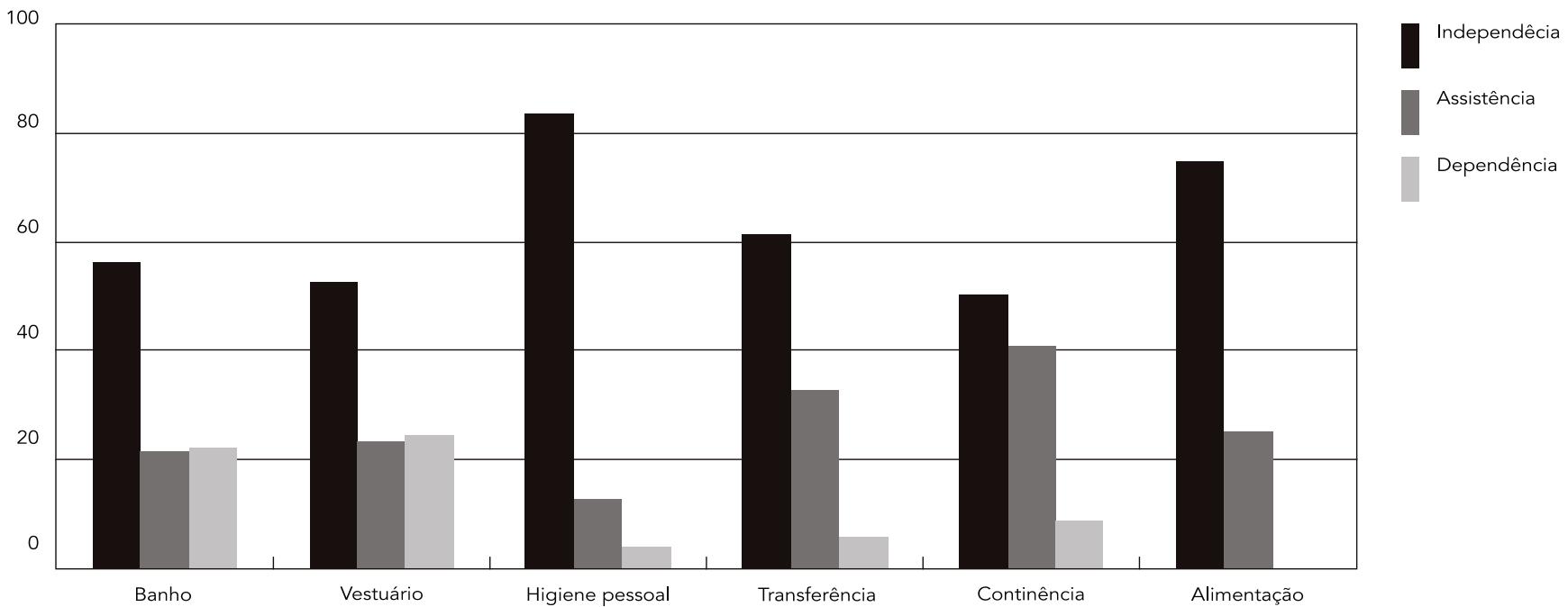


Figura 2

Estratégias de coping utilizadas pelos idosos em face das dificuldades funcionais.

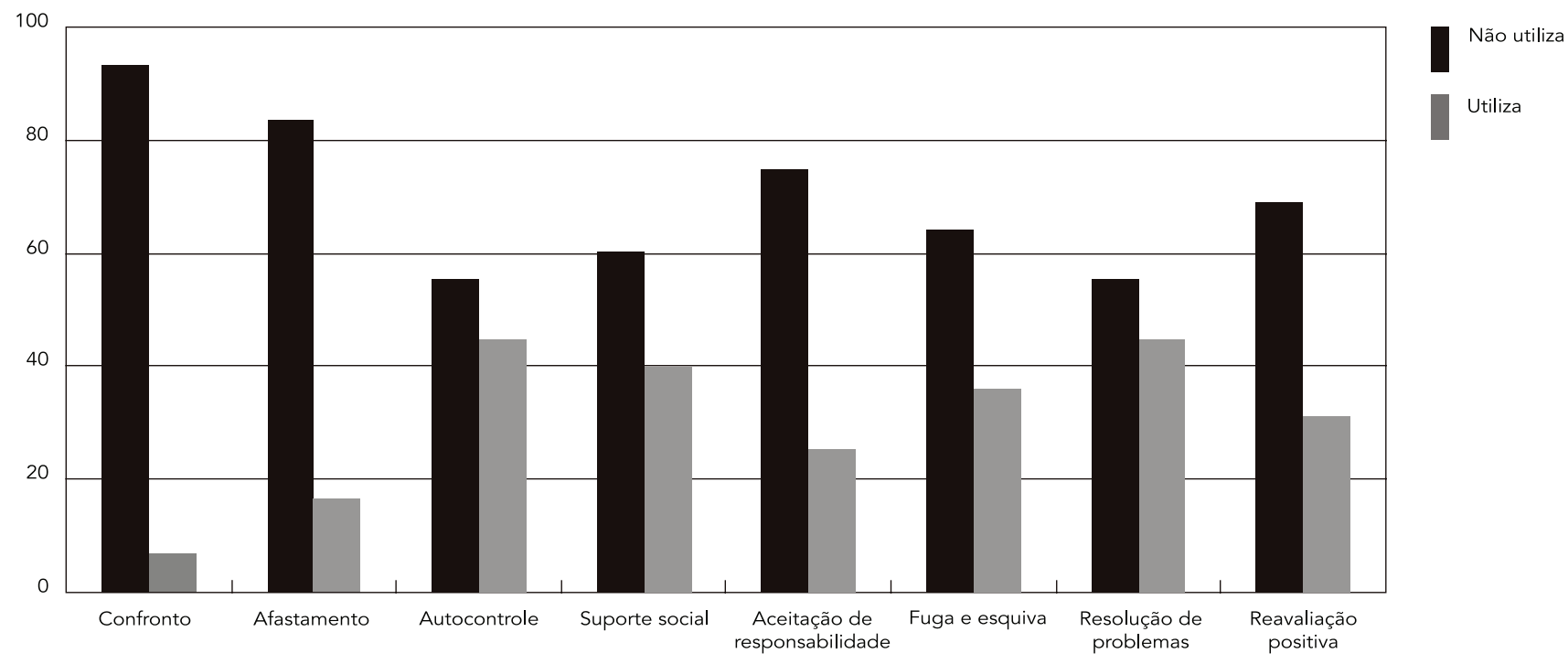

Tabela 1

Associação entre estratégias de coping e sintomas depressivos.

\begin{tabular}{|c|c|c|c|c|}
\hline & \multicolumn{3}{|c|}{ Sintomas depressivos } & \multirow[t]{2}{*}{ Valor de $p$} \\
\hline & Mínimo & Leve a moderado & Grave & \\
\hline \multicolumn{5}{|l|}{ Confronto } \\
\hline Não utiliza & 92,2 & 95,1 & 90,9 & 0,742 \\
\hline Utiliza & 7,8 & 4,9 & 9,1 & \\
\hline \multicolumn{5}{|l|}{ Afastamento } \\
\hline Não utiliza & 78,4 & 90,2 & 81,8 & 0,299 \\
\hline Utiliza & 21,6 & 9,8 & 18,2 & \\
\hline \multicolumn{5}{|l|}{ Autocontrole } \\
\hline Não utiliza & 52,9 & 53,7 & 72,7 & 0,511 \\
\hline Utiliza & 47,1 & 46,3 & 27,3 & \\
\hline \multicolumn{5}{|l|}{ Suporte Social } \\
\hline Não utiliza & 47,1 & 73,2 & 72,7 & 0,027 \\
\hline Utiliza & 52,9 & 26,8 & 27,3 & \\
\hline \multicolumn{5}{|c|}{ Aceitação de responsabilidade } \\
\hline Não utiliza & 72,5 & 75,6 & 81,8 & 0,850 \\
\hline Utiliza & 27,5 & 24,4 & 18,2 & \\
\hline \multicolumn{5}{|l|}{ Fuga e esquiva } \\
\hline Não utiliza & 64,7 & 65,9 & 54,5 & 0,801 \\
\hline Utiliza & 35,3 & 34,1 & 45,5 & \\
\hline \multicolumn{5}{|c|}{ Resolução de problemas } \\
\hline Não utiliza & 52,9 & 56,1 & 63,6 & 0,847 \\
\hline Utiliza & 47,1 & 43,9 & 36,4 & \\
\hline \multicolumn{5}{|c|}{ Reavaliação positiva } \\
\hline Não utiliza & 66,7 & 68,3 & 81,8 & 0,682 \\
\hline Utiliza & 33,3 & 31,7 & 18,2 & \\
\hline
\end{tabular}


Associação entre estratégias de coping e sexo.

\begin{tabular}{|c|c|c|c|}
\hline & \multicolumn{2}{|c|}{ Sexo } & \multirow[t]{2}{*}{ Valor de $p$} \\
\hline & Masculino & Feminino & \\
\hline \multicolumn{4}{|l|}{ Confronto } \\
\hline Não utiliza & 83,3 & 94,5 & 0,188 \\
\hline Utiliza & 16,7 & 5,5 & \\
\hline \multicolumn{4}{|l|}{ Afastamento } \\
\hline Não utiliza & 83,3 & 83,5 & 1,000 \\
\hline Utiliza & 16,7 & 16,5 & \\
\hline \multicolumn{4}{|l|}{ Autocontrole } \\
\hline Não utiliza & 58,3 & 54,9 & 1,000 \\
\hline Utiliza & 41,7 & 45,1 & \\
\hline \multicolumn{4}{|l|}{ Suporte social } \\
\hline Não utiliza & 75,0 & 58,2 & 0,354 \\
\hline Utiliza & 25,0 & 41,8 & \\
\hline \multicolumn{4}{|c|}{ Aceitação de responsabilidade } \\
\hline Não utiliza & 66,7 & 75,8 & 0,493 \\
\hline Utiliza & 33,3 & 24,2 & \\
\hline \multicolumn{4}{|l|}{ Fuga e esquiva } \\
\hline Não utiliza & 50,0 & 65,9 & 0,342 \\
\hline Utiliza & 50,0 & 34,1 & \\
\hline \multicolumn{4}{|c|}{ Resolução de problemas } \\
\hline Não utiliza & 33,3 & 58,2 & 0,129 \\
\hline Utiliza & 66,7 & 41,8 & \\
\hline \multicolumn{4}{|c|}{ Reavaliação positiva } \\
\hline Não utiliza & 58,3 & 70,3 & 0,508 \\
\hline Utiliza & 41,7 & 29,7 & \\
\hline
\end{tabular}

ficativos na associação entre idade e estratégias utilizadas. Além disso, também não se verificou associação entre as estratégias e o estado civil, nem na relação com a percepção dos idosos sobre sua saúde.

\section{Discussão}

No que diz respeito à amostra de idosos institucionalizados, os resultados desta pesquisa comprovam a tendência de estudos nesta área, em que a participação masculina raramente ultrapassa os 20\% 23. Quanto à percepção da própria saúde, o resultado evidenciado pela população de residentes em asilos demonstrou pequena diferença em relação à população idosa geral brasileira. Em estudo desenvolvido por Lima-Costa et al. 24, 10,5\% dos idosos percebiam sua saúde como ruim. No presente estudo, $11,7 \%$ classificaram-na como tal .

Em relação às atividade de vida diária, estudo desenvolvido por Lucena et al. 12 demonstra, por intermédio do índice de Katz, as tarefas de banho e vestuário como as que mais exigem assistência ou dependência por parte dos idosos institucionalizados, com $48,6 \%$ e $35,9 \%$ respectivamente. Guedes \& Silveira 11, utilizando a escala de Barthel, encontraram, na atividade de banho, o mais elevado número de dependência. Outros altos índices de dependência, parcial ou total, apresentados pela pesquisa, referem-se a atividades de vestuário, higiene pessoal e micção. Embora o presente estudo tenha mostrado resultados similares ao evidenciar elevado nível de dependência ou assistência nas atividades de vestuário e banho, a tarefa de continência apresentou-se como a de maior necessidade de auxílio.

As estratégias de enfrentamento mais utilizadas pela amostra deste estudo foram a de autocontrole e resolução de problemas. A estratégia de autocontrole refere-se à tentativa de regulação de sentimentos e ações, enquanto a estratégia de resolução de problemas indica o esforço para a administração ou solução do problema 25 . No entanto, para analisar a ade- 
Associação entre estratégias de coping e anos de estudo.

\begin{tabular}{|c|c|c|c|c|}
\hline & \multicolumn{3}{|c|}{ Anos de estudo } & \multirow[t]{2}{*}{ Valor de $\mathrm{p}$} \\
\hline & Analfabeto & 1 a 4 & 5 ou mais & \\
\hline \multicolumn{5}{|l|}{ Confronto } \\
\hline Não utiliza & 91,7 & 76,0 & 100,0 & 0,001 \\
\hline Utiliza & 8,3 & 24,0 & 0 & \\
\hline \multicolumn{5}{|l|}{ Afastamento } \\
\hline Não utiliza & 66,7 & 84,0 & 86,4 & 0,298 \\
\hline Utiliza & 33,3 & 16,0 & 13,6 & \\
\hline \multicolumn{5}{|l|}{ Autocontrole } \\
\hline Não utiliza & 33,3 & 56,0 & 59,0 & 0,200 \\
\hline Utiliza & 66,7 & 44,0 & 41,00 & \\
\hline \multicolumn{5}{|l|}{ Suporte social } \\
\hline Não utiliza & 58,3 & 68,0 & 57,6 & 0,965 \\
\hline Utiliza & 41,7 & 32,0 & 42,4 & \\
\hline \multicolumn{5}{|c|}{ Aceitação de responsabilidade } \\
\hline Não utiliza & 83,3 & 76,0 & 72,7 & 0,893 \\
\hline Utiliza & 16,7 & 24,0 & 27,3 & \\
\hline \multicolumn{5}{|l|}{ Fuga e esquiva } \\
\hline Não utiliza & 58,3 & 80,0 & 59,0 & 0,443 \\
\hline Utiliza & 41,7 & 20,0 & 41,0 & \\
\hline \multicolumn{5}{|c|}{ Resolução de problemas } \\
\hline Não utiliza & 66,7 & 60,0 & 51,5 & 0,229 \\
\hline Utiliza & 33,3 & 40,0 & 48,5 & \\
\hline \multicolumn{5}{|c|}{ Reavaliação positiva } \\
\hline Não utiliza & 66,7 & 72,0 & 68,2 & 0,282 \\
\hline Utiliza & 33,3 & 28,0 & 31,8 & \\
\hline
\end{tabular}

quação da estratégia escolhida pelos idosos que apresentam dificuldade funcional, é importante considerar o contexto em que o evento estressor ocorre, tendo em vista que determinada estratégia pode ser eficaz para algumas situações, mas não para outras 26 .

Não obstante isso, os oito fatores propostos por Lazarus \& Folkman 14 foram evidenciados na amostra deste estudo, o que corrobora resultados de pesquisas anteriores que revelam associação entre os diferentes estilos de coping, podendo apresentar ou não o predomínio de uma determinada estratégia 27.

Pinheiro et al. 28 enfatizam que, apesar de as pesquisas na área de coping terem crescido na última década, questões conceituais e metodológicas sobre o constructo permanecem em discussão. Um fator que ainda apresenta divergências em diferentes estudos é quanto às categorias de estratégias de coping. Algumas estratégias podem ser consideradas efetivas em determinado ambiente, mas não se mostrarem adequadas em outra situação.
Segundo Ryan-Wenger 29, alguns autores sustentam que as estratégias de coping centradas no problema são mais adaptativas do que as centradas na emoção, uma vez que permitiriam a mudança da situação. Porém, o autor defende que as estratégias são inerentemente neutras, só podendo ser avaliadas como positivas ou negativas conforme a situação e os resultados obtidos. Logo, levando-se em consideração a importância da dimensão situacional no processo de coping, instrumentos de medidas desenvolvidos para situações específicas poderiam ser mais efetivos na avaliação das estratégias de enfrentamento 30,31 .

O estudo das estratégias de coping tem mostrado relações significativas com algumas variáveis sócio-demográficas, como gênero ${ }^{30}$. Em estudo desenvolvido por Folkman \& Lazarus 32, que investigou as estratégias de coping diante de eventos ocupacionais, os homens utilizaram mais estratégias focadas no problema do que as mulheres. Em pesquisa desenvolvida com adolescentes, essa tendência também foi evidencia- 
da, com jovens do sexo feminino utilizando mais intensamente as estratégias de coping focadas na emoção ${ }^{33}$. Todavia, no presente estudo, apesar de os homens terem utilizado em maior grau a estratégia de resolução de problemas e as mulheres a estratégia de autocontrole, não foi evidenciada associação significativa.

Em pesquisa cujo objetivo era identificar os estilos de coping utilizados por 60 idosos, sendo 30 de um grupo-controle e 30 com doença de Alzheimer, observou-se que a escolaridade pode ter exercido influência positiva no enfrentamento de situação de estresse, auxiliando o indivíduo a eleger o foco do problema 26 . No presente estudo, evidenciou-se que idosos com menor escolaridade utilizaram em maior grau a estratégia de confronto, representando esforços agressivos de alteração da situação, podendo envolver características de hostilidade e risco, o que pode acarretar prejuízo no manejo do problema.

Endler \& Parker 34 sugerem que as estratégias de coping também podem ser influenciadas pela depressão. Em pesquisa desenvolvida por Galdino 27 , os idosos com sintomas depressivos apresentaram maior tendência a utilizar estratégias de coping focadas na emoção em situações de estresse.

\section{Resumo}

O objetivo deste estudo é verificar as estratégias de enfrentamento utilizadas pelos idosos em face das dificuldades funcionais e a existência de associação entre o uso das estratégias e variáveis como sintomas depressivos, sexo, escolaridade, idade, estado civil e percepção da saúde. Participaram do estudo 103 idosos institucionalizados, que apresentavam necessidade de assistência em no mínimo uma atividade de vida diária. O delineamento foi transversal, com a utilização dos seguintes instrumentos: Ficha de Dados Pessoais, Escala de Atividades da Vida Diária, Breve Entrevista Internacional de Neuropsiquiatria Modificada, MiniExame do Estado Mental, Inventário de Estratégias de Coping $e$ Escala de Depressão Geriátrica. As estratégias de enfrentamento mais utilizadas pela amostra foram a de autocontrole e resolução de problemas. Encontraram-se associações significativas entre as variáveis escolaridade e depressão. Os resultados da pesquisa sugerem que a avaliação das estratégias utilizadas em face de eventos estressores pode auxiliar na análise da situação e na adequação das estratégias escolhidas, ajudando também em processos de mudança no contexto clínico.

Saúde do Idoso Institucionalizado; Depressão; Idoso Fragilizado
Os resultados deste estudo demonstraram associação entre nível mínimo de sintomas depressivos e uso da estratégia de suporte social. Por sua vez, a estratégia de suporte social pode apresentar-se como uma estratégia focada tanto no problema, na busca por suporte informativo, quanto na emoção, em caso de suporte emocional. Para poder categorizar a estratégia de suporte emocional entre os dois subtipos, seria necessário avaliar o intuito do uso da estratégia. Para Sharrer \& Ryan-Wenger 35, a categorização em estratégias focadas no problema e focadas na emoção implica um conhecimento prévio da intenção ou motivação no uso da estratégia.

Portanto, a avaliação das estratégias de enfrentamento utilizadas pode trazer benefícios num contexto clínico, tanto para os idosos institucionalizados, quanto para a população geral.

O presente estudo verificou estratégias de coping utilizadas para lidar com dificuldades funcionais, no entanto, em face de qualquer situação estressante, lança-se mão de diferentes estratégias. Assim, investigar as estratégias utilizadas diante de diferente contextos e eventos de vida pode auxiliar profissionais de saúde na análise de situações, no entendimento da maneira de enfrentamento e em possível intervenção para a adequação da estratégia escolhida.

\section{Colaboradores}

A. S. Vivan contribuiu na elaboração e delineamento do projeto, pesquisa de campo, análise de dados e redação do artigo. I. I. L. Argimon participou de todas as etapas.

\section{Agradecimentos}

À Coordenação de Aperfeiçoamento de Pessoal de Nível Superior (CAPES) pelo financiamento. 


\section{Referências}

1. Instituto Brasileiro de Geografia e Estatística. Projeção da população do Brasil para o período 19802050: revisão 2004. Rio de Janeiro: Instituto Brasileiro de Geografia e Estatística; 2004.

2. Melo I, Brito M. 181.586.030 brasileiros. Zero Hora 2004; 31 ago.

3. Busse EW, Blazer D. Psiquiatria geriátrica. 2a Ed. Porto Alegre: Editora Artes Médicas; 1999.

4. Ramos L, Rosa, T, Oliveira, Z, Medina, M, Santos, F. Profile of the elderly residents in São Paulo, Brazil: results from a household survey. Rev Saúde Pública 1993; 27:87-94.

5. Ramos LR. Fatores determinantes do envelhecimento saudável em idosos residentes em centro urbano: Projeto Epidoso, São Paulo. Cad Saúde Pública 2003; 19:793-8.

6. Fiedler MM, Peres KG. Capacidade funcional e fatores associados em idosos do sul do Brasil: um estudo de base populacional. Cad Saúde Pública 2008; 24:409-15.

7. Guedea MT, Albuquerque FJ, Tróccoli BT, Noriega JA, Seabra MA, Guedea RL. Relação do bem-estar subjetivo, estratégias de enfrentamento e apoio social em idosos. Psicol Reflex Crit 2006; 19:301-8.

8. Silva JC. Velhos ou idosos? A Terceira Idade 2003 14:94-111.

9. Ribeiro R, Silva A, Modena C, Fonseca M. Capacidade funcional e qualidade de vida de idosos. Estudos Interdiscip Envelhec 2002; 4:85-96.

10. Karsch UM. Idosos dependentes: famílias e cuidadores. Cad Saúde Pública 2003; 19:861-6.

11. Guedes JM, Silveira, RC. Análise da capacidade funcional da população geriátrica institucionalizada na cidade de Passo Fundo-RS. Revista Brasileira de Ciências do Envelhecimento Humano 2004; 1:10-21.

12. Lucena NM, Guerra RO, Lucena AB, Silva CF, Nascimento RQ. Análise da capacidade funcional em uma população geriátrica institucionalizada em João Pessoa. Fisioterapia Brasil 2002; 3:164-9.

13. Pereira A, Freitas C, Mendonça C, Marçal F, Souza J, Noronha JP, et al. Envelhecimento, estresse e sociedade: uma visão psiconeuroendocrinológica. Ciências \& Cognição, 2004; 1:34-53.

14. Lazarus R, Folkman S. Stress, appraisal and coping. New York: Springer Publishing Company; 1984.

15. Folkman S. Personal control and stress and coping processes: a theoretical analysis. J Pers Soc Psychol 1984; 46:839-52.

16. Zeidner M, Saklofske D. Adaptive and maladaptive coping. In: Zeidner M, Endler NS editors. Handbook of coping. New York: Wiley; 1996. p. 505-31.

17. Kraaij V, Pruymboom E, Garnefski N. Cognitive coping and depressive symptoms in the elderly: a longitudinal study. Aging Ment Health 2002; 6:275-81.

18. Katz S. Assessing self-maintenance: activities of daily living, mobility and instrumental activities of daily living. J Am Geriatr Soc 1983; 31:721-7.

19. Amorim P. Mini International Neuropsychiatric Interview (MINI): validação de entrevista breve para diagnóstico de transtornos mentais. Rev Bras Psiquiatr 2000; 22:106-15.
20. Folstein MF, Folstein SE, McHugh PR. Mini-mental state: a pratical method for grading the cognitive state of patients for the clinician. J Psychiatr Res 1975; 12:189-98.

21. Savóia MG, Santana P, Mejias NP. Adaptação do Inventário de Estratégias de Coping de Folkman e Lazarus para o português. Psicol USP 1996; 7: 183-201.

22. Yesavage JA, Brink TL, Rose TL, Lum O, Huang V, Adey MB, et al. Development and validation of a geriatric depression screening scale: a preliminary report. J Psychiatr Res 1983; 17:37-49.

23. Davim R, Torres G, Dantas S, Lima V. Estudo com idosos de instituições asilares no município de Natal/RN: características socioeconômicas e de saúde. Rev Latinoam Enferm 2004; 12:518-24.

24. Lima-Costa MF, Barreto S, Giatti L. Condições de saúde, capacidade funcional, uso de serviços de saúde e gastos com medicamentos da população idosa brasileira: um estudo descritivo baseado na Pesquisa Nacional por Amostra de Domicílios. Cad Saúde Pública 2003; 19:735-43.

25. Folkman S, Lazarus R. Manual for the ways of coping questionnaire. Mountain View: Consulting Psychologists Press; 1988.

26. Souza J, Chaves E, Caramelli P. Coping em idosos com doença de Alzheimer. Rev Latinoam Enferm 2007, 15:93-9.

27. Galdino J. Ansiedade, depressão e coping em idosos [Dissertação de Mestrado]. São Paulo: Escola de Enfermagem, Universidade de São Paulo; 2000.

28. Pinheiro F, Tróccoli B, Tamayo M. Mensuração de coping no ambiente ocupacional. Psicol Teor Pesqui 2003; 19:153-8.

29. Ryan-Wenger NA. Children, coping and the stress of illness: a synthesis of the research. J Soc Pediatr Nurses 1996; 1:126-39.

30. Parkes K. Coping, negative affectivity and the work environment: additive and interactive predictors of mental health. J Appl Psychol 1990, 75:399-409.

31. Clark K, Bormann C, Cropanzano R, James K. Validation evidence for three coping measures. J Pers Assess 1995; 65:434-55.

32. Folkman S, Lazarus R. An analysis of coping in a middle-aged community sample. J Health Soc Behav 1980; 25:229-44.

33. Li C. The roles of sex, gender and coping in adolescent depression [Dissertação de Mestrado]. New York: Department of Psychology, St. John's University; 2000.

34. Endler N, Parker J. Multidimensional assessment of coping: a critical evaluation. J Pers Soc Psychol 1990; 58:844-54.

35. Sharrer V, Ryan-Wenger N. A longitudinal study of age and gender differences of stressors and coping: strategies in school aged children. J Pediatr Health Care 1995; 9:123-30.

Recebido em 18/Mar/2008

Versão final reapresentada em 06/Ago/2008

Aprovado em 29/Ago/2008 